\title{
A Dynamic Technique of Oral Rehabilization Using Fixed Prosthesis and CSC Telescopic Denture for Severe Advanced Periodontitis with Secondary Occlusal Traumatism - A Long-term Case Report
}

\author{
Guey-Lin Hou
}

\section{ABSTRACT}

Clinical reports of many literatures had documented that meticulous nonsurgical periodontal therapy (NSPT) and combined with periodontal prosthesis application might not only improve periodontal parameters, but also provide effective reduction of microbiological organisms associated with periodontal pathogen.

\begin{abstract}
A 54-year old female individual visited with chief complains of gingival bleeding and mobile teeth, and recurrent periodontal abscess over \# 13, \# 11, \# 21, and \# 22. Periapical radiographs disclosed moderate periodontal bone loss on the total remained teeth of maxilla and mandible arches. A diagnosis of generalized severe advanced periodontitis (SAP) with secondary occlusal traumatism (SOT) was established. This present case was to document the treatment of a 6 years longitudinally retrospective evaluation of case affected SAP with SOT using treatment of the combination of periodontal and perioprosthetic design. Results showed that the remarkable improvement of periodontal tissues, reconstruction of posterior bite occlusion and recovery of esthetic appearance.
\end{abstract}

It can be concluded that the Sandwich's technique in treating patients affected SAP with SOT is an effective and valuable option.

Keywords: Sandwich's technique, CSCTD, Oral rehabilization, SAP, SOT.
Published Online: December 07, 2021

ISSN: $2684-4443$

DOI: $10.24018 /$ ejdent.2021.2.6.119

\section{Guey-Lin Hou*}

Former professor, Department of Periodontics, and Graduate Institute of Dental Science, Department of Periodontal Prosthesis, Kaohsiung Medical University, Kaohsiung City, Taiwan.

Former professor, Department of Periodontal \& Prosthetic Therapeutic Center, Chang Gung Memorial Hospital, Kaohsiung Medical Center, Taiwan.

(e-mail: houg12001@yahoo.com.tw)

*Corresponding Author

\section{INTRODUCTION}

Initial periodontitis can be assessed using probing of clinical periodontal attachment and radiographic periodontal bone. Former reports documented that the initial stage of the marginal periodontitis with small changes or crest bone loss is not accurately estimated by conventional radiography [1[3]. The understanding the periodontal epidemiology associated with different types of periodontal diseases, such as juvenile periodontitis (JP), chronic periodontitis (CP), and aggressive periodontitis (AP) has been from retrospective study via clinical diagnosis among individuals reporting the teaching hospitals [4]-[6]. In addition, other reports related to the distribution, prevalence, and periodontal bone loss rates based on the long-term radiographic evaluation of clinically healthy cases were also documented [7], [8]. Only limited studies associated with the extent periodontal bone destruction versus ages, degree and location in patients affected periodontitis have been reported [9], [10].

There is well known that little or limited reports regarding bilateral occlusal bite collapse and the extent alveolar bone loss alteration due to neglect and not known the endanger teeth of severity early loss of permanent teeth affected advanced severe adult periodontitis (SAP) with secondary occlusal traumatism (SOT). It is necessary for cases involved SAP with SOT of full mouth oral rehabilitation affected with bilateral occlusal bite collapse and the extent alveolar bone loss alteration.

The purpose of present study was to investigate the therapeutic outcomes of full mouth oral rehabilitation affected with bilateral occlusal bite collapse and the extent alveolar bone loss alteration in a case affected SAP with SOT.

\section{CASE REPORT}

A 54-year old female individual visited our office with chief complains of gingival bleeding and mobile teeth, and recurrent periodontal abscess over \# 13, \# 11, \# 21, and \# 22. According to the patient's complain, she had mobile teeth of upper and lower arches, especially located on the \# 13, \# 11, \# 21, and \# 22. In addition, the other complaints of occlusal interference were also noted.

Oral examination indicated that maxillary missing teeth were located on the \#17- \#15, \#12, and \#24 - \#26, where the mandibular missing teeth located on the \# 35- \#37 and \# 45, \#46 with ill-fitted clasped removable partial denture were also found, respectively.

Assessments of periodontal parameters of maxillary and 
mandibular teeth included gingival index (GI) [11], plaque index (PLI) [12], probing pocket depth (PPD), and clinical attachment level (CAL), were recorded at baseline and every six months until the end of the study. Periapical radiographs disclosed moderate periodontal bone loss on the total remained teeth of maxilla and mandible arches (Fig. 1). In addition, severe advanced bone loss and SOT were noted on the \# 12, respectively. A diagnosis generalized SAP with SOT was established (Fig. 2a, 2b, and 2c).
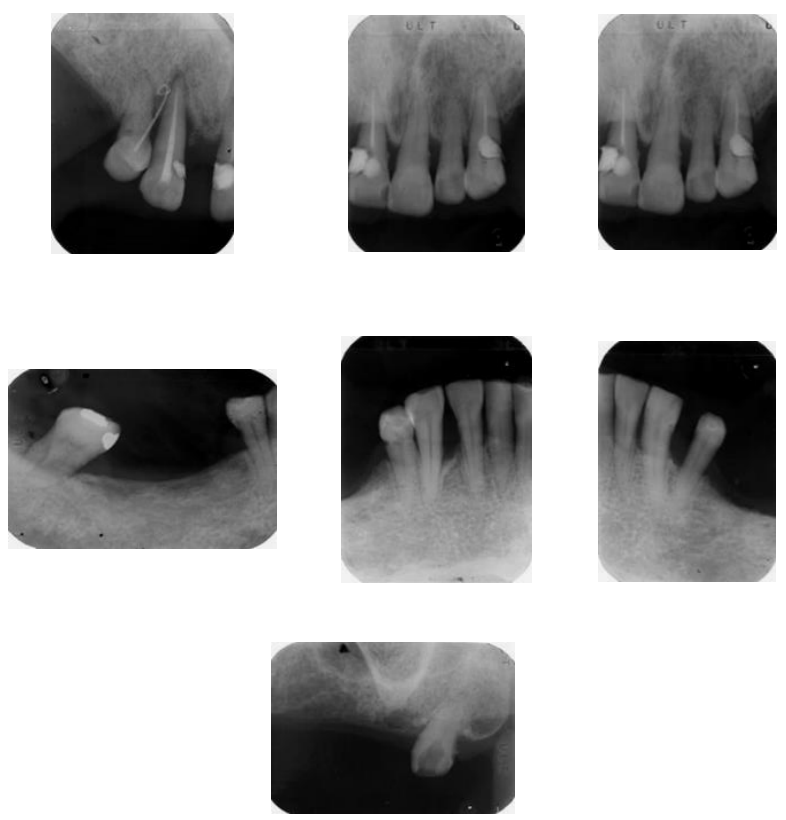

Fig. 1. Radiographs disclosed moderate and severe periodontal bone loss on the remained teeth of maxilla and mandible arches at baseline. $(2009 / 3 / 6)(2009 / 3 / 6)$.

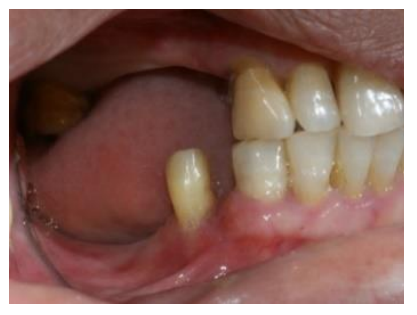

$2 \mathrm{a}$

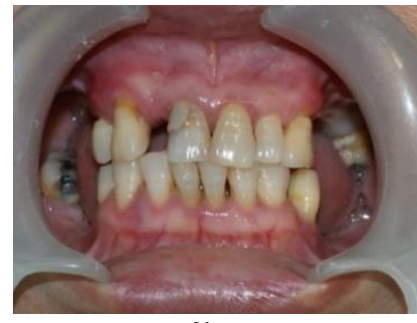

$2 \mathrm{~b}$

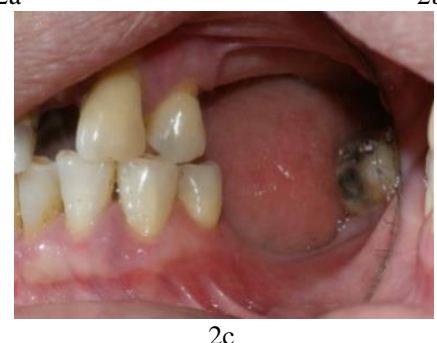

$2 \mathrm{c}$

Fig. 2. Clinical appearance of oral remained teeth in both maxilla and mandible arches at baseline.

Treatment sequences including removed Ill-fitted clasp removable partial denture, oral hygiene instruction, basic periodontal treatment including subgingival scaling, curettage, basic occlusal adjustment, root planning. Personal and professional oral hygiene educations are the basic priority, following subgingival scaling, root planning and periodontal pocket irrigation with Chlorhexidine glouconate (1\%). Construction of maxillary fixed prosthesis of \#11, \#21, and \#22, and application of inner crowns at \#14, \# 13, \# 23, and \# 27. Finally, removable prosthesis of crown and sleevecoping telescopic denture was applied at \#17- \#12 and \#23\#27), respectively (Fig. 3a, 3b, 3c, and 3d).

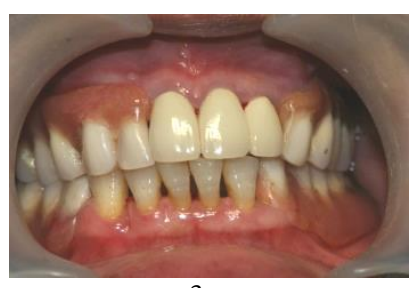

$3 \mathrm{a}$

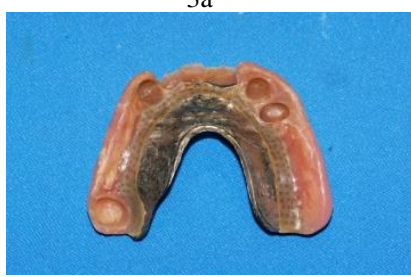

$3 \mathrm{c}$

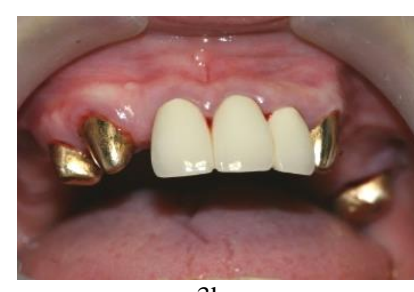

$3 \mathrm{~b}$

$3 d$

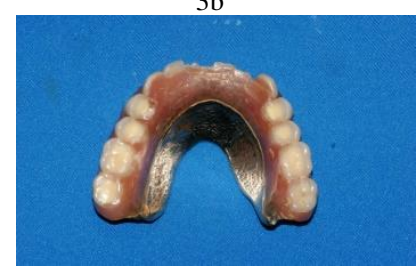

Fig. 3. Removable prosthesis of crown and sleeve-coping telescopic denture (c, d) was applied at \#17-\#12 and \#23-\#27(b, d), respectively. (abutments on the \#13, \#14, \#23, and \# 27) (b, d).

Application of lower mandibular inner crowns (Fig. 4a) at \#34, \#33, \#44, and \#47, and followed by the removable crown and sleeve-coping telescopic denture crowns (Fig. 4b).

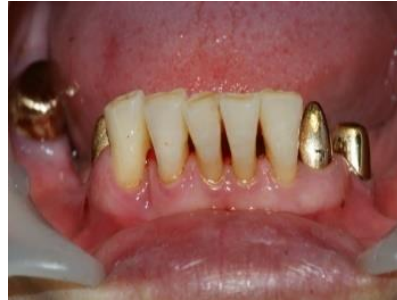

a

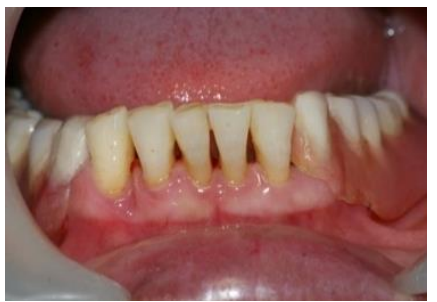

b
Fig. 4. Application of lower mandibular inner crowns (a) at \#34, \#33, \#44, and \#47, and followed by the removable crown and sleeve-coping telescopic denture crowns (b).

Fig. 5a and 5b showed the clinical appearance of maxillary and mandibular periodontal prosthesis of both fixed crowns at \#11, \#21, and \#22, inner crowns (Fig. 5a), and CSCTD (Fig. 5b) at the upper and lower arches, respectively.

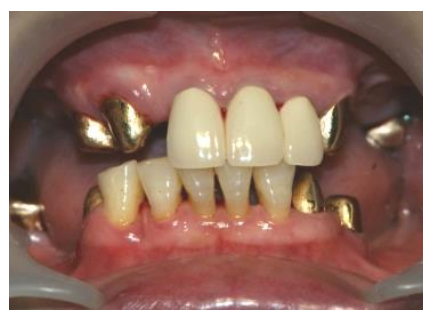

a

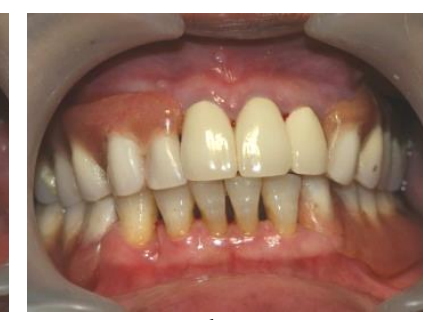

Fig. 5. Buccal views showed the clinical appearance of maxillary and mandibular periodontal prosthesis of both fixed crowns at \#11,\#21, and \#22, inner crowns (a), and CSCTD (b) at the upper and lower arches, respectively.

Radiographic image analysis (Fig. 6a, 6b, 6c) indicated the present case affected SAP with SOT demonstrated that prominent improvement of periodontal bony defects before periodontal treatment after periodontal and prosthetic treatments. In addition, remarkable healings of alveolar crests, lamina dura, and bone fills were noted at the baseline of angular bone defects after using the treatment of 
Sandwich's technique including NSPT, fixed prosthesis and CSCTD as compared to those radiographic images at the baseline, respectively.

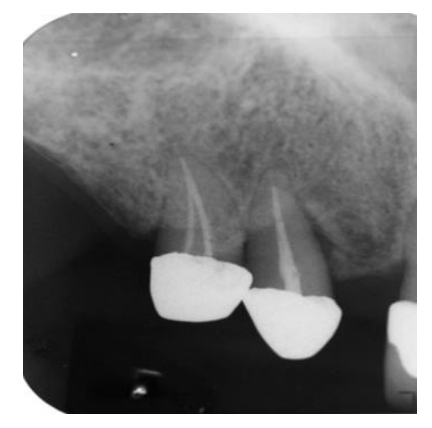

a

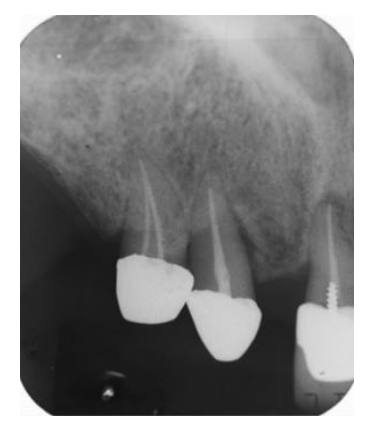

b

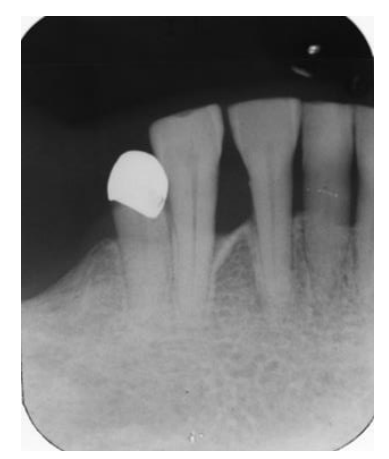

$\mathrm{c}$

Fig. 6. Periapical radiographs indicated that remarkable healing of angular bony defects on the total abutment teeth of maxilla and mandible arches after NSPT and CSCTD perioprosthetic therapy. (2015/2/20).

Periapical radiographs disclosed moderate periodontal bone loss on the total remained teeth of maxilla and mandible arches (Fig. 6a, 6b, and 6c; 2015/2/20).

\section{DISCUSSION}

\section{A. Clinical Findings after Periodontal Treatment}

Clinical symptoms and signs disappeared almost completely within 2-4 weeks and showed remarkable and excellent healing in both gingival and periodontal tissue afterward until the end of study 5 years later. Leon and Vogel [13] demonstrated that ultrasonic debridement was more effective than hand scaling in class II and III molar furcation involvement (FI) at reducing spirochetes and motile rods. It has been documented that most favorable modality in periodontal therapy in the removal of all soft and hard deposits on root surfaces exposed by SAP [14], [15].

\section{B. Periodontal Bone Fills}

Andreasen [16] suggested that even the loss of periodontal ligament, up to $2 \mathrm{~mm}$ in width around the root surface, may be repaired by a new periodontal attachment. Our former reports [17]-[19] also confirmed the fact that remarkable healing of periodontal bone occurred after using non-surgical periodontal therapy, immobilization of teeth affected severe periodontal bone loss with SOT, and periodontal prosthesis after mid-term (3-5years) and long-term (5-39 years) [20] evaluations. The present case affected SAP with SOT demonstrated that prominent improvement of periodontal parameters, periodontal tissues, and remarkable alveolar crests, lamina dura, and bone fills (Fig. 6a, 6b) located at the angular bone defects using NSPT, fixed prosthesis and CSCTD.

\section{Clinical application of CSCTD}

The effect of immobilization on teeth with mobile and poor bony support using Sandwich's technique of provisional prosthesis (PP), NSPT, and CSCTD seems to promote remarkable healing of soft and hard periodontal tissue. This finding is consistent and supported with earlier investigators. [21]-[25] We, thus, concluded that the sequential use of Sandwich's technique [20] included PP, NSPT, and CSCTD appeared to be a valuable alternative for preserving the compromised teeth that served as an abutment necessary for restoring occlusal function.

\section{Radiographic Analysis}

Radiographic image analysis indicated the present case affected SAP with SOT demonstrated that prominent improvement of periodontal bony defects before periodontal treatment. In addition, remarkable healings of alveolar crests, lamina dura, and bone fills (Fig. 6a, 6b, 6c) were noted at the baseline of angular bone defects after using the treatment of Sandwich's technique including NSPT, fixed prosthesis and CSCTD as compared to those radiographic images at the baseline data, respectively.

\section{REFERENCES}

[1] Lang NP, Hill RW. Radiographs in periodontics. J Clin Periodontol, 1997; 4: 16-28.

[2] Kornman KA. Nature of periodontal disease: Assessment and diagnosis. J Periodont Res, 1987; 22: 192-204.

[3] Goodson JM, Haffajee AD, Socransky SS. The relationship between attachment level loss and alveolarbone loss. J Clin Periodontol, 1984 11: 348-359

[4] Boyle WS jr.,Via WF, MacFacc WT Jr. Radiographic analysis of alveolar crest height and age. J Periodontol, 1973; 44: 236-242.

[5] O' Conner TW, Biggs NL. Interproximal bony contours. J Periodontol, 1965; 35:326-330.

[6] Selikowitz HS, Sheiham A, Albert D, Williams GM. Retrospective longitudinal study of the rate of alveolar bone loss in humans using bitewing radiograps. J Clin Periodont, 1981; 8: 431-438.

[7] Suomi JD, Plumo J, Barbano JP. A comparative study of radiographs and pocket measurements in periodontal disease evaluation. $J$ Periodontol, 1968; 39: 311-315.

[8] Manson JD, Nicholson K. The distribution of bone defects in chronic periodontitis. J Periodontol, 1974; 45: 88-92.

[9] Abander JM, Brown LJ, Genco RJ, Loe H. Clinical classification of periodontitis in adolescents and young adults. J Periodontol, 1997; 68 : 545-555.

[10] Hou GL, Tsai CC. Relationship between periodontal furcation involvement and molar cervical enamel projection. J Periodontol, 1987; 58: 715-721.

[11] Loe H, Silness L. Periodontal disease in pregnancy. I. Prevalence and severity. Acta Odontol Scand, 1963; 21: 533-551.

[12] Silness L, Loe H. Periodontal disease in pregnancy. II. Correlation between oral hygiene and periodontal condition. Acta Odontol Scand, 1964; 22:121-135.

[13] Leon L, Vogel R. A comparison of the effectiveness of hand scaling and ultrasonic debridement in furcations as evaluated by differential darkfieldmicroscopy. J Periodontol, 1987; 58: 86-94.

[14] Muller HP, Eger T, Langer DE. Management of furcation-involved teeth - A retrospective analysis. J Clin Periodont 1995; 22: 911-917.

[15] Hamp SE, Nyman S, Lindhe J. Periodontal treatment of multirooted teeth results after 5 years. J Clin Periodont, 1975; 2: 126-135.

[16] Andreasen JO. Interrelation between alveolar bone and periodontal ligament repair after replantation of mature permanent incisors in monkeys. J Periodont Res, 1981; 16: 228-235. 
[17] Hou GL. A long-term study of oral rehabilitation for the treatment of severe advanced periodontitis with second occlusal traumatism using intentional replantation, fixed prosthesis and perioprosthetic design of CSCTD. Advances in Dentistry \& Oral Health, 2021; 4(5): 1-5.

[18] Hou GL. Differences of annual radiographic alveolar bone loss rates of anterior and posterior teeth of individuals affected with secondary occlusal traumatism between with and without perioprosthetic therapy. Advances in Dentistry \& Oral Health, 2021; 14(4): 1-5.

[19] Hou GL. Annually and cumulative radiographic alveolar bone loss rates using digital scanning image for the periodontal disease groups before and after periodontal treatment. European $J$ Dent and Oral Health 2021; 2(3): 1-5.

[20] Hou GL, Hou LT. Therapeutic outcomes using the Sandwich's technique in treating severe advanced periodontitis with secondary occlusal traumatism: A long-term study for 5.1-39 years. Intern $J$ Dentisry and Oral Health 2019;5(7):76-85.

[21] Yakisove IL. Crown and sleeve-coping retainers for removable partial denture. J Prosthet Dent, 1966; 16: 1069-1085.

[22] Hou GL, Tsai CC, Weisgold AS. Treatment of molar furcation involvement using root separation and a crown and sleeve-coping telescopic denture - A longitudinal study. J Periodontol, 1999; 70: 1098-1109.

[23] Yalisove IL, Diez JB. Telescope Prosthetic Therapy - Biomechanics of the crown and sleeve-coping prosthesis, $1^{\text {st }}$ ed., Chapter 5, Philadelphia; Strickly, 1977; pp. 65-82.

[24] Hou GL. Survival rates of CSC telescopic abutments of severe advanced periodontitis with secondary occlusal traumatism using periodontal and prosthetic therapies. A long-term study of case series for 5.1-19.3 years. Advances in Dent \& Oral Health, 2020; 13(1):250256.

[25] Hou GL. A long-term evaluation of bone levels using digital scanning radiographic image analysis on the CSC telescopic abutments in severe advanced periodintitis affected secondary occlusal Trauma- tism. Advances in Dent \& Oral Health, 2021; 14(5): 1-5. 